\title{
Limb Salvage and Functional Outcomes following Free Tissue Transfer for the Treatment of Recalcitrant Diabetic Foot Ulcers
}

\author{
Jocelyn Lu, BS ${ }^{1}$ Michael V. DeFazio, MD² Chrisovalantis Lakhiani, MD² Michel Abboud, BS ${ }^{1}$ \\ Morgan Penzler, BS ${ }^{1}$ Tammer Elmarsafi, DPM, MBBCh² Paul J. Kim, DPM, MS ${ }^{1}$ \\ Christopher E. Attinger, MD² Karen K. Evans, MD²
}

\footnotetext{
${ }^{1}$ Department of Plastic Surgery, Georgetown University School of Medicine, Washington, DC

2 Department of Plastic Surgery, Center for Wound Healing, MedStar Georgetown University Hospital, Washington, DC
}

J Reconstr Microsurg 2019;35:117-123.
Address for correspondence Karen K. Evans, MD, Department of Plastic Surgery, Center for Wound Healing and Hyperbaric Medicine, MedStar Georgetown University Hospital, 3800 Reservoir Road, NW, 1-PHC, Washington, DC 20007

(e-mail: Karen.K.Evans@gunet.georgetown.edu).

\begin{abstract}
Keywords

- diabetic foot ulcer

- free tissue transfer

- limb salvage

Background Recent evidence documenting high success rates following microvascular diabetic foot reconstruction has led to a paradigm shift in favor of more aggressive limb preservation. The primary aim of this study was to examine reconstructive and functional outcomes in patients who underwent free tissue transfer (FTT) for recalcitrant diabetic foot ulcers (DFUs) at our tertiary referral center for advanced limb salvage.

Methods Between June 2013 and June 2016, 29 patients underwent lower extremity FTT for diabetic foot reconstruction by the senior author (K.K.E.). In all cases, microsurgical reconstruction was offered as an alternative to major amputation for the management of recalcitrant DFUs. Overall rates of flap survival, limb salvage, and postoperative ambulation were evaluated. The lower extremity functional scale (LEFS) score was used to assess functional outcomes after surgery.

Results Overall rates of flap success and lower limb salvage were 93 and 79\%, respectively. Flap failure occurred in two patients with delayed microvascular compromise. Seven patients in this series ultimately required below-knee amputation secondary to recalcitrant infection $(n=5)$, intractable pain $(n=1)$, and limb ischemia $(n=1)$. The average interval between FTT and major amputation was 8 months ( $r, 0.2-15$ months). Postoperative ambulation was confirmed in 25 patients (86\%) after a mean final follow-up of 25 months ( $r, 10-48$ months). The average LEFS score for all patients was 46 out of 80 points ( $r, 12-80$ points), indicating the ability to ambulate in the community with some limitations.

Conclusion FTT for the management of recalcitrant DFUs is associated with high rates of reconstructive success and postoperative ambulation. However, several patients will eventually require major amputation for reasons unrelated to ultimate flap survival. These data should be used to counsel patients regarding the risks, functional implications, and prognosis of microvascular diabetic foot reconstruction.
\end{abstract}

received

March 5, 2018

accepted after revision

June 27, 2018

published online

August 12, 2018
Copyright $\odot 2019$ by Thieme Medical Publishers, Inc., 333 Seventh Avenue, New York, NY 10001, USA. Tel: +1(212) 584-4662.
DOI https://doi.org/

10.1055/s-0038-1667363. ISSN 0743-684X. 
Advances in microvascular surgery have redefined the way plastic surgeons approach lower extremity reconstruction in the modern era. Wounds that once would have condemned a limb for amputation are now salvageable and functional. Despite these advances, many patients with complex lower extremity wounds and ischemic limbs continue to undergo amputation. This is largely due to a misconception in the medical community that patients with multiple medical problems, such as diabetes mellitus, hypertension, peripheral vascular disease (PVD), and coronary artery disease, are poor candidates for advanced lower extremity reconstruction. ${ }^{1}$ Many subscribers to this misconception believe that amputation may afford a safer and more reliable alternative for highly comorbid patients.

However, lower extremity amputation (LEA) carries significant postoperative morbidity, and societal costs associated with this procedure are not necessarily less when compared with limb salvage. ${ }^{2,3}$ It is well known that soon after LEA in diabetic patients, the contralateral limb may be threatened with the same fate. ${ }^{4,5}$ These patients are less likely to ambulate, ${ }^{6}$ and overall postamputation mortality is higher in this group of patients than in nondiabetics. ${ }^{7}$ As a group, diabetic patients with multiple comorbidities are ill equipped to tolerate the increased physical demands that accompany major amputation, and are even less able to tolerate bilateral limb loss. ${ }^{8-10}$ The fundamental reason for emphasis on limb salvage and preservation of limb length is that the increased energy of ambulation in amputees results in greater cardiopulmonary demands. In conjunction with concomitant medical comorbidities and decreased physiologic reserve, this increased demand leads to higher mortality rates among amputees following surgery.

Microsurgical advancements have expanded the use of free tissue transfer (FTT) in reconstructive surgery. In patients with large defects of the lower extremity in which local or regional flaps are inadequate, FTT offers a well-contoured soft tissue envelope that can withstand a dynamic workload with minimal disruption of tendon glide. ${ }^{11}$ FTT also offers well-vascularized soft tissue coverage from outside the zone of injury and can serve to aid in perfusion of the distal lower extremity, especially in patients with preexisting vascular disease. ${ }^{12}$

At our tertiary referral center for complex wound management, we are often presented with the challenge of attempting limb salvage in highly comorbid, diabetic patients with advanced systemic disease who face major LEA as the alternative. The primary objective of this study was to evaluate the reconstructive and functional outcomes of patients who underwent FTT for the management of recalcitrant diabetic foot ulcers (DFUs).

\section{Methods}

\section{Patient Demographics}

A single-center retrospective review was conducted for all consecutive patients who underwent FTT for diabetic foot reconstruction between June 2013 and June 2016. All procedures were performed by the senior author (K.K.E.) Data were collected and analyzed in accordance with an Institu- tional Review Board-approved protocol for patients who presented with recalcitrant DFUs and were considered candidates for major LEA. Recalcitrant ulcers were defined as those that failed previous attempts at both nonoperative wound care and surgical management, including serial debridement and various reconstructive modalities (i.e., primary closure, skin graft with/without dermal regenerate template application, local-regional flaps). Demographic data, reconstructive characteristics, outcomes, and postoperative ambulatory status were documented for all cases.

\section{Patient Selection and Perioperative Management Protocol}

Patients were selected for microsurgical intervention based on their overall health status and functional prognosis. In general, patients with medically optimized comorbidities who were ambulatory, preoperatively, and were able to tolerate a prolonged operation under general anesthesia, along with a protracted recovery, were considered ideal candidates for advanced limb salvage. In addition, patients were also chosen based on their willingness and ability to comply with rigorous postoperative rehabilitation and physical therapy regimens. Patients who presented with sepsis secondary to infected DFUs were medically managed and stabilized before being considered for inclusion into this cohort. Patients who were preoperatively nonambulatory and/or suffered from intractable pain were felt to be better candidates for major LEA, and were thus excluded from this cohort.

All patients in this study were treated in an acute setting requiring inpatient hospital stay. Pertinent laboratories including complete blood count with differential, comprehensive metabolic panel, hemoglobin A1c, prealbumin, albumin, prothrombin time, partial thromboplastin time, international normalized ratio, and a hypercoagulability panel were obtained at the time of admission.

Preoperative evaluation with plain radiographs and/or computed tomography/magnetic resonance imaging was used to assess extent of wounds and degree in infection. All patients also underwent lower extremity vascular evaluation with endovascular angiography by the vascular surgery team. Patients who required reperfusion via balloon angioplasty or stenting angioplasty were typically put on a regimen of Plavix (Bristol-Myers Squibb Co., New York City, NY) and aspirin, and reconstruction with FTT was generally performed approximately 5 to 10 days after reperfusion. Orthopedic consults were placed for patients requiring bony stabilization prior to reconstruction. Intraoperative cultures were obtained following each debridement and were used to guide sensitivity-directed antibiotic therapy as well as the timing of definitive reconstruction. Local wound care with either wet-to-dry dressing changes or vacuum-assisted closure was provided in the interim between debridements. Patients with osteomyelitis underwent bone biopsies along with aggressive debridement to healthy, bleeding bone. Any infected hardware or foreign objects were removed, dead space was filled with well-vascularized tissue (i.e., fasciocutaneous or muscle flaps), and a prolonged course (i.e., 6 weeks) of culture-driven antibiotics was administered. 
For all patients, the decision to proceed with FTT was guided by the presence of negative cultures, absence of clinical signs of infection, and adequate response to local wound care. Dual vein anastomosis to the deep venous system was preferred for lower extremity FTT. When adequate deep veins were unavailable, superficial counterparts were incorporated.

Following successful FTT, flaps were monitored for 5 days in an intensive care unit setting, through a combination of clinical examination (i.e., color, temperature, turgor, capillary refill, bleeding) and Doppler monitoring of arterial and venous signals. For flaps without a cutaneous paddle and/or those in which a reliable venous signal could not be obtained with a hand-held Doppler, an implantable Cook Doppler probe (Cook Medical, Bloomington, NJ) was used to provide continuous venous monitoring during the early postoperative period. Flaps demonstrating signs of arterial and/or venous insufficiency were taken back to the operating room for emergent exploration. Patients with uncomplicated flaps were started on a progressive dangling protocol, beginning postoperative days 5 to 6 , and were discharged once the flap was deemed stable after 15 minutes of continuous gravitational dependence (i.e., typically day 10 or 11). Beginning 4 weeks postoperatively, patients were enrolled in a graduated physical rehabilitation program. Weight bearing in a controlled ankle movement walker boot was allowed 4 to 6 weeks after FTT, depending on the location of the flap. Patients were also seen by a pedorthotist at this time for the fabrication of customized shoes and/or inserts.

\section{Outcomes Analysis}

Primary outcomes included overall flap survival, rates and timing of major amputation, and postoperative ambulatory status. Functional outcomes after surgery were evaluated using the lower extremity functional scale (LEFS) score. The LEFS evaluates patients' ability to perform 20 various ambulatory activities (i.e., performing work around the house, putting on socks/shoes, walking up/down stairs, running, etc.). Patients rate their abilities on a scale from 0 to 4 , with 0 indicating extreme difficulty or inability to perform tasks, and 4 indicating no difficulty with the task. All responses are summated, giving the total LEFS score. Administration of the survey was conducted at least 9 months after FTT to assess outcomes of the reconstruction after patients had adequate time to recover and regain ambulatory capacity. Subgroup analysis of functional outcomes in patients with successful limb salvage versus those with below-knee amputation (BKA) was performed. The Fisher's exact test was performed to evaluate correlation between patient comorbidities and primary outcomes.

\section{Results}

Over a 3-year period, 29 patients (18 males and 11 females) with a mean age of 57 years $(r, 24-71$ years) underwent microvascular FTT for the reconstruction of recalcitrant DFUs, with an average follow-up of 25 months ( $r, 10-48$ months). The average body mass index (BMI) was $31 \mathrm{~kg} / \mathrm{m}^{2}$ $\left(r, 23-45 \mathrm{~kg} / \mathrm{m}^{2}\right)$, and mean glycated hemoglobin (HbA1c) was $7.5 \%(r, 5-11 \%)$. Comorbidities in this population included hypertension ( $n=26)$, PVD $(n=21)$, chronic kidney disease $(n=11)$, regular tobacco use $(n=5)$, and chronic obstructive pulmonary disease $(n=4)$. Fisher's exact test did not find any of the above comorbidities to significantly increase risk of either flap loss $(p>0.05)$ or BKA $(p>0.05)$. Patients presented with at least one DFU affecting the ankle $(n=13)$, plantar foot $(n=6)$, heel $(n=7)$, Achilles tendon $(n=7)$, and forefoot $(n=4)$. A majority of defects were reconstructed with anterolateral thigh $(n=14)$, vastus lateralis $(n=11)$, radial forearm $(n=2)$, or latissimus dorsi $(n=1)$ flaps. One patient, who underwent resection of an infected first metatarsophalangeal joint, underwent reconstruction with a medial femoral condyle osteocutaneous flap. Patients required an average of 3.4 debridements ( $r$, 1-8 debridements) prior to FTT, with FTT reconstruction occurring on average 21 days after admission ( $r, 5-48$ days). Of the 29 patients, 25 had vacuum-assisted closure, and the remaining four patients had wet-to-dry dressings.

Demographic data, comorbidities, defect location, and flap selection are presented in -Tables $\mathbf{1}$ and $\mathbf{2}$.

Outcomes and complications following FTT are presented in -Table 3. The overall flap success rate was $93 \%$ in this series. Of the 29 free flaps performed for diabetic foot reconstruction, 4 patients (14\%) required take backs to the operating room for microvascular compromise $(n=3)$ and hemorrhage $(n=1)$. Two of these take backs resulted in flap salvage. The first case of flap demise was caused by delayed venous thrombosis on postoperative day 8 that was deemed unsalvageable in the operating room. The patient was treated conservatively with negative pressure wound therapy and a dermal substitute (Integra LifeSciences, Plainsboro, NJ) until

Table 1 Patient demographics and comorbidities in diabetic limb salvage patients

\begin{tabular}{|c|c|}
\hline & Patients $(n=29)$ \\
\hline \multicolumn{2}{|l|}{ Demographic characteristics } \\
\hline \multicolumn{2}{|l|}{ Age, y } \\
\hline Mean (range) & $57.3(24.5-71.3)$ \\
\hline \multicolumn{2}{|l|}{ Gender } \\
\hline Male (\%) & $18(62)$ \\
\hline Female & $11(38)$ \\
\hline \multicolumn{2}{|l|}{ BMI, $\mathrm{kg} / \mathrm{m}^{2}$} \\
\hline Mean (range) & $30.9(23.2-45.1)$ \\
\hline \multicolumn{2}{|l|}{ Comorbidities (\%) } \\
\hline Diabetes mellitus & $29(100)$ \\
\hline Hypertension & $26(89.7)$ \\
\hline COPD & $4(13.8)$ \\
\hline Chronic kidney disease & 11 (37.9) \\
\hline Peripheral vascular disease & $21(72.4)$ \\
\hline Current smoker & $5(17.2)$ \\
\hline
\end{tabular}

Abbreviations: BMI, body mass index; COPD, chronic obstructive pulmonary disease. 
Table 2 Wound and reconstructive characteristics in diabetics undergoing lower extremity free tissue transfer

\begin{tabular}{|c|c|}
\hline & Patients $(n=29)$ \\
\hline \multicolumn{2}{|l|}{ Wound characteristics } \\
\hline \multicolumn{2}{|l|}{ Location (\%) } \\
\hline Ankle & $13(35.1)$ \\
\hline Plantar foot & $6(16.2)$ \\
\hline Heel & 7 (18.9) \\
\hline Achilles tendon & 7 (18.9) \\
\hline Forefoot & $4(10.8)$ \\
\hline \multicolumn{2}{|l|}{ Infection status (\%) } \\
\hline Soft tissue infection & $9(31)$ \\
\hline Osteomyelitis & $20(69)$ \\
\hline \multicolumn{2}{|l|}{ Vascular inflow (\%) } \\
\hline Three-vessel runoff (no PVD) & $8(28)$ \\
\hline \multicolumn{2}{|l|}{ PVD patients } \\
\hline$\leq 1$ vessel runoff & $16(55)$ \\
\hline$\geq 2$ vessel runoff & $5(17)$ \\
\hline \multicolumn{2}{|l|}{ Reconstructive characteristics } \\
\hline \multicolumn{2}{|l|}{ Debridements prior to FTT } \\
\hline Mean (range) & $3.4(1-8)$ \\
\hline \multicolumn{2}{|l|}{ Time from presentation to FTT, mo } \\
\hline Mean (range) & $0.8(0.2-2.7)$ \\
\hline No. of flaps (\%) & $29(100)$ \\
\hline \multicolumn{2}{|l|}{ Flap type (\%) } \\
\hline Anterolateral thigh & $14(48.3)$ \\
\hline Vastus lateralis & $10(34.5)$ \\
\hline Radial forearm & $2(6.9)$ \\
\hline Latissimus dorsi & $2(6.9)$ \\
\hline Medial femoral condyle & $1(3.4)$ \\
\hline
\end{tabular}

Abbreviation: FTT, free tissue transfer.

a skin graft could be successfully placed. The second case of flap loss occurred in a patient with a previous ankle arthrodesis and severe PVD, leading to single-vessel flow to the lower extremity. The patient desired aggressive efforts at limb salvage for occupational concerns, thus FTT transfer was attempted. The artery was anastomosed end to side along with a single deep vena comitantes (no other veins, deep or superficial, were suitable). Intraoperatively, the flap was noted to have somewhat sluggish flow, but no clear sign of imminent flap failure. By the sixth postoperative day, it was evident that the flap was nonviable, and we felt a welldesigned BKA would provide this particular patient with the best opportunity for early return to functional activity and ambulation with a prosthesis while minimizing the morbidity of a protracted reconstructive course.

In total, seven patients required major LEA during the follow-up period, resulting in an overall limb salvage rate of $76 \%$ for this series. Indications for major amputation
Table 3 Reconstructive outcomes and complications after free tissue transfer for diabetic limb salvage

\begin{tabular}{|c|c|}
\hline & Patients $(n=29)$ \\
\hline \multicolumn{2}{|l|}{ Reconstructive outcomes (\%) } \\
\hline Flap success & $27(93)$ \\
\hline \multicolumn{2}{|l|}{ Operative take backs (\%) } \\
\hline Microvascular compromise & $3(10.3)$ \\
\hline Hemorrhage & $1(3.4)$ \\
\hline Limb salvage (\%) & $22(76)$ \\
\hline \multicolumn{2}{|l|}{ Complications } \\
\hline \multicolumn{2}{|l|}{ Dehiscence (\%) } \\
\hline Donor site & $2(6.9)$ \\
\hline Recipient site & $1(3.4)$ \\
\hline \multicolumn{2}{|l|}{ Seroma (\%) } \\
\hline Donor site & $0(0)$ \\
\hline Recipient site & $1(3.4)$ \\
\hline \multicolumn{2}{|l|}{ Hematoma (\%) } \\
\hline Donor site & $2(6.9)$ \\
\hline Recipient site & $0(0)$ \\
\hline \multicolumn{2}{|l|}{ Surgical site infection (\%) } \\
\hline Donor site & $4(13.8)$ \\
\hline Recipient site & $1(3.4)$ \\
\hline Below-knee amputation (\%) & $7(24.1)$ \\
\hline \multicolumn{2}{|l|}{ Indications for BKA } \\
\hline Underlying hardware infection & $4(57.1)$ \\
\hline Soft tissue infection & $1(14.3)$ \\
\hline Chronic intractable pain & $1(14.3)$ \\
\hline $\begin{array}{l}\text { Unreconstructable PVD } \\
\text { after failed FTT }\end{array}$ & $1(14.3)$ \\
\hline \multicolumn{2}{|l|}{ Time from FTT to BKA, mo } \\
\hline Mean (range) & $8(0.2-15)$ \\
\hline
\end{tabular}

Abbreviations: BKA, below-knee amputation; FTT, free tissue transfer; PVD, peripheral vascular disease.

included persistent hardware infection $(n=4)$, recalcitrant soft tissue infection $(n=1)$, and chronic intractable pain $(n=1)$. The average interval between FTT and subsequent amputation was $8 \pm 5.2$ months ( $r, 0.2-15$ months). Other postoperative complications included four (14\%) patients with wound dehiscence, four (14\%) patients with surgical site infection, and one patient (3\%) requiring debulking.

In addition, 25 (86\%) patients were ambulatory postoperatively. Patients began bearing weight on the lower extremity at an average of $2 \pm 1.3$ months $(r, 0.7-5.1)$ after FTT. Average time to regain full weight-bearing status was $3 \pm 1.5$ months $(r, 1.4-6.5)$ post-FTT. In comparing functional outcomes for patients with successful limb salvage to those underwent BKA, both subgroups were equivalent with regard to rates ( 86 vs. $86 \%, p>0.05$ ) and timing (3.4 vs. 2.7 months, $p>0.05$ ) of ambulation. 
Table 4 Functional outcomes following successful limb salvage versus below knee amputation

\begin{tabular}{|c|c|c|c|}
\hline & $\begin{array}{l}\text { Limb salvage } \\
\text { cohort } \\
(n=22)\end{array}$ & $\begin{array}{l}\text { BKA cohort } \\
(n=7)\end{array}$ & p-Value \\
\hline \multicolumn{4}{|l|}{ Outcomes } \\
\hline $\begin{array}{l}\text { Independent } \\
\text { ambulation } \\
\text { (\%) }\end{array}$ & $19(86)$ & $6(86)$ & $>0.05$ \\
\hline \multicolumn{4}{|c|}{ Ambulatory timeline } \\
\hline \multicolumn{4}{|c|}{ Time to initiate weight bearing, mo } \\
\hline $\begin{array}{l}\text { Mean } \\
\text { (range) }\end{array}$ & $2.1(0.7-5.1)$ & $1.2(0.7-1.4)$ & $>0.05$ \\
\hline \multicolumn{4}{|c|}{ Time to full weight bearing, mo } \\
\hline $\begin{array}{l}\text { Mean } \\
\text { (range) }\end{array}$ & $3.4(1.4-6.5)$ & $2.7(1.5-4.4)$ & $>0.05$ \\
\hline \multicolumn{4}{|c|}{ Lower extremity functional scale score } \\
\hline $\begin{array}{l}\text { Mean } \\
\text { (range) }\end{array}$ & $45(12-80)$ & $49(22-80)$ & $>0.05$ \\
\hline
\end{tabular}

Abbreviation: BKA, below-knee amputation.

The LEFS survey was administered to patients at a mean time of $19 \pm 11$ months ( $r$, 3.5-36 months) after FTT, with an average score of $46 \pm 22(r, 12-80$; - Table 3$)$. The average LEFS score was $45 \pm 22.7(r, 12-80)$ in the limb salvage cohort and $49 \pm 21.6(r, 22-80)$ in the BKA cohort. Ambulatory outcomes and LEFS scores are presented in - Table 4. Data points in the LEFS were also separated into low-demand (i.e., performing household activities, putting on shoes and socks, etc.) and high-demand activities (i.e., running, making sharp turns while running, etc.). After FTT, patients scored on average 2.8 out of 4 points in low-demand activities, and 1.3 out of 4 points in high-demand activities. Outcomes for specific data points in the LEFS are presented in - Fig. 1.

\section{Discussion}

Reconstruction of complex foot wounds in diabetic patients presents a challenging problem. With the incidence of diabetes increasing in the United States, treatment for this disease and related complications have accumulated direct costs of more than 116 billion dollars. ${ }^{13}$ Of that, at least one-third is allocated to treating DFUs, with the mean cost of treatment per ulcerative episode ranging from $\$ 4,595$ to $\$ 15,792 .{ }^{13,14}$ These contribute significantly to the global health care expenditure. Unfortunately, progression of DFUs to major LEA has demonstrated poor outcomes, with 5-year mortality rates as high as 4 to $82 \%{ }^{1,10}$ In addition, the risk of contralateral LEA reaches $\sim 50 \%$ by 2 years after index LEA. ${ }^{5}$ Major LEAs in diabetic patients with comorbid systemic illnesses may lead to unrecoverable morbidity and inevitable mortality. While previous studies have shown that FTT is a viable option for the management of recalcitrant DFUs, ${ }^{1,15-19}$ data regarding the correlation among free flap success, functional outcomes, and ultimate limb salvage are limited.

Overall rates of free flap success and lower limb salvage in the present series were 93 and $76 \%$, respectively. A prior study by $\mathrm{Oh}$ et al reported 121 cases of free flaps for diabetic lower extremities with a $91.7 \%$ flap survival rate and limb salvage rate of $84.9 \% .^{1}$ Despite disparities in Eastern and Western populations (i.e., most notably a BMI of 30.9 in our series compared with 23.17 reported previously ${ }^{1}$ ), flap success and limb salvage rates are quite similar. This series

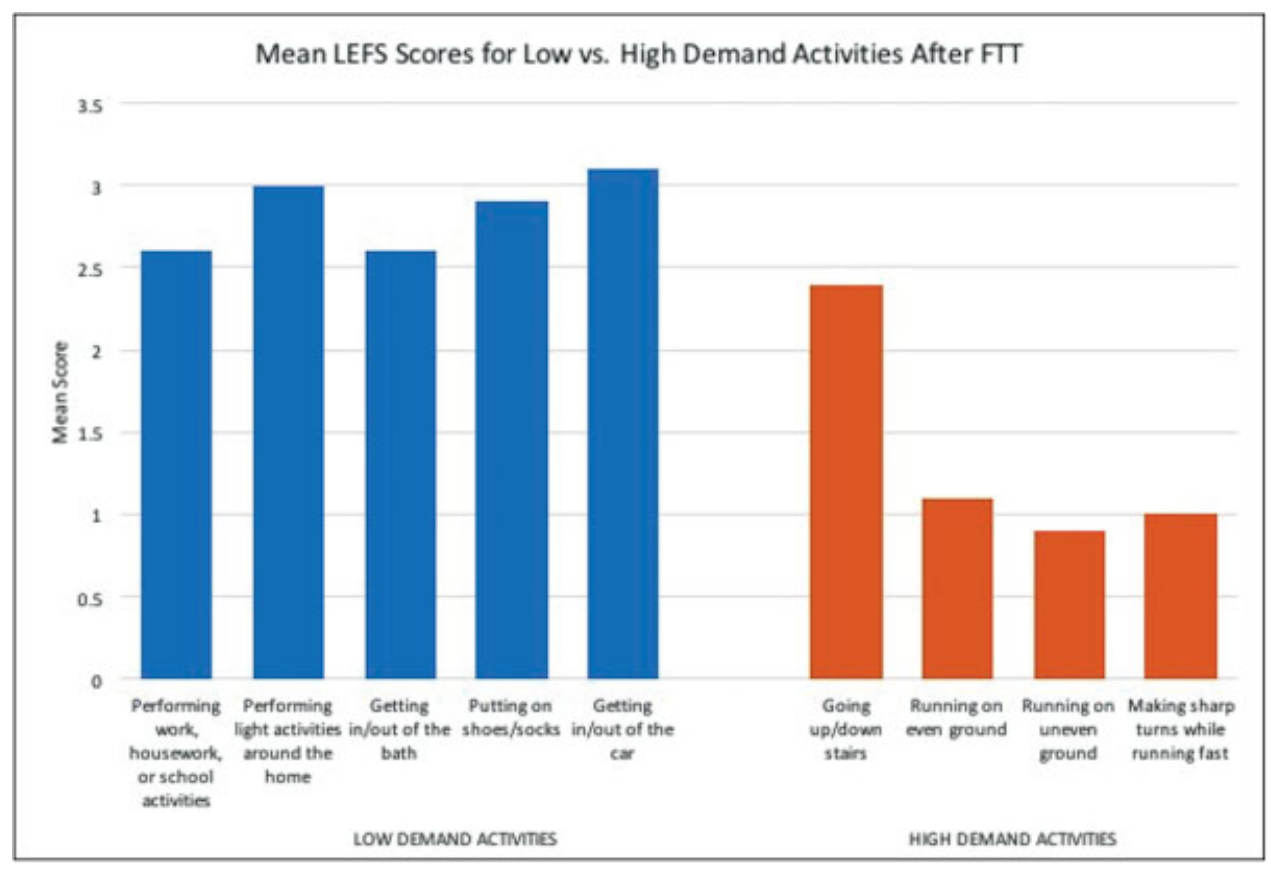

Fig. 1 Mean LEFS scores after FTT for diabetic limb salvage. Higher mean scores for low-demand versus high-demand activities indicate that patients are more likely to return to independent ambulation and basic functionality after successful free flap reconstruction. FTT, free tissue transfer; LEFS, lower extremity functional scale. 
supports the notion that FTT in the Western population is a viable option for the treatment of recalcitrant DFUs, where major LEA is the alternative.

Although we observed a high flap success rate (93\%) in our cohort, seven patients (24\%) in this study eventually went on to receive a BKA after a mean of 8 months, postoperative. Interestingly, only one patient in this series required BKA due to failure of the flap itself, whereas, six patients underwent BKAs for recalcitrant soft tissue infection $(n=1)$, hardware infection $(n=4)$, or intractable pain $(n=1)$. This suggests that other extrinsic factors beyond free flap survival may play a more significant role in ultimate limb prognosis. Patients with a history of persistent infection (especially in the setting of implants/hardware), or chronic refractory pain may not be optimal candidates for aggressive limb salvage, regardless of overall health status. Prior studies evaluating FTT for limb salvage of the diabetic lower extremity also report higher flap survival rates (92-96\%) than limb salvage rates (83-87\%), but the indications for major LEA in those series were often undisclosed. ${ }^{1,15,19,20}$

Functional outcomes after FTT to the diabetic lower extremity have not been extensively evaluated. Our institution previously published a meta-analysis of 50 articles, including 1,079 flaps for lower extremity reconstruction, demonstrating that functional outcomes are infrequently and inconsistently reported in the literature. ${ }^{21}$ In a systematic review of 528 diabetic patients from 18 studies, Fitzgerald O'Connor et al found a mean ambulatory rate of $76 \%$ after FTT for chronic nonhealing ulcers, although no validated or quantifiable measures were indicated in their study. ${ }^{15}$ The $86 \%$ ambulation rate in this series supports the favorable outcomes documented in previous reports. In addition, we used the LEFS survey, a validated, patient-derived, metric to assess functional outcomes after FTT. Our average LEFS score of 46 falls within the "limited community ambulator" category ${ }^{22}$-suggesting that diabetics who undergo advanced lower extremity reconstruction with FTT are able to recover and lead functional, ambulatory lives. Our institution recently published an article of LEFS survey responses for 101 free flaps for lower extremity reconstruction, concluding that patients are able to independently ambulate and function in the community after FTT. ${ }^{23}$ To our knowledge, our current study is the first to publish objective functional outcomes data following FTT in the diabetic limb salvage population. We encourage future studies to implement validated functional outcomes surveys and quality-of-life measures, such as the LEFS and Short Form 36 , respectively, to objectively compare outcomes between institutions and populations.

Functional outcomes in diabetics after BKA have historically been bleak, with reports of only 34 to $49 \%$ of patients regaining the ability to ambulate postoperatively. ${ }^{6,24}$ Results from our series show a much higher ambulation rate of $86 \%$ in our BKA cohort. In addition, the average LEFS score in these patients was comparable to that of the limb salvage cohort. We utilize a multidisciplinary approach including plastic surgeons, vascular surgeons, podiatric surgeons, orthopedic surgeons, and infectious disease specialists, to determine if a patient would achieve better function with FTT or a BKA. In general, we choose BKA for the highest functional demand patients who have already attempted surgeries at limb salvage, and we approach the BKA surgery as a functional surgery and not a failure. For these patients, we work closely with prosthetists and physical therapists to ensure patients have well-designed prostheses and are undergoing the appropriate rehabilitation to regain independent ambulation after LEA.

In evaluating specific LEFS data points post-FTT, we found that, unsurprisingly, patients were able to perform lowdemand activities with less difficulty than high-demand activities. FTT to the lower extremity does not come without risks, but can be expected to provide most patients the ability to resume basic daily activities without sacrificing independence or functionality around 3 months, postoperatively. This is especially pertinent to the diabetic population, in whom limb functionality and postoperative ambulatory capacity may directly correlate with patient survival. ${ }^{8,25}$

Flap choice was determined by many factors including specific defects and goals unique to each patient. The vast majority of flaps used in this cohort were derived from the lateral circumflex femoral system of the thigh. In defects with large amounts of dead space, muscle flaps (i.e., vastus lateralis) were preferred, whereas fasciocutaneous flaps were preferred for resurfacing of the dorsal foot. In addition, free muscle flaps were preferred over fasciocutaneous flaps in obese patients due to the ability of muscle to atrophy after reconstruction, allowing for more normal lower extremity contour and ability better fit into footwear.

This study is limited by its retrospective nature and small sample size, which limits the ability of statistical analysis to detect significant differences in outcome between limb salvage and major amputation cohorts. In addition, a longer mean follow-up would be ideal to assess patient outcomes and functionality at 5 to 10 years after reconstruction. This cohort was also subject to selection bias, as only the patients with the highest probability of successful FTT were chosen to undergo aggressive attempts at lower limb salvage. This inevitably leads to somewhat inflated flap success and limb salvage rates in this difficult population. These data, along with other preliminary studies, provide a strong foundation and rationale for FTT as an alternative to amputation in the treatment of DFUs. ${ }^{15}$ Welldesigned, prospective studies evaluating long-term limb salvage rates, ambulation, and mortality after FTT in the diabetic population are needed to optimize patient selection as well as confirm functional advantages, quality of life, and survival benefits over major LEA.

\section{Conclusion}

This study demonstrates promising reconstructive and functional outcomes following FTT for recalcitrant DFUs. Despite high rates of free flap success, however, a significant percentage of patients may eventually require amputation for reasons unrelated to ultimate flap survival. These findings should be used to counsel patients regarding the risks, functional implications, and prognosis of microvascular diabetic foot reconstruction. With proper screening, patient 
selection, and medical optimization using a multidisciplinary approach, FTT can serve as an effective means for limb salvage and enable return to ambulation in the diabetic population with complex lower extremity wounds.

\section{Financial Disclosures}

None of the authors have any financial disclosures, commercial associations, or any other conditions posing a conflict of interest to report.

\section{References}

1 Oh TS, Lee HS, Hong JP. Diabetic foot reconstruction using free flaps increases 5-year-survival rate. J Plast Reconstr Aesthet Surg 2013;66(02):243-250

2 Gupta SK, Veith FJ, Ascer E, Flores SA, Gliedman ML. Cost factors in limb-threatening ischaemia due to infrainguinal arteriosclerosis. Eur J Vasc Surg 1988;2(03):151-154

3 Mackey WC, McCullough JL, Conlon TP, et al. The costs of surgery for limb-threatening ischemia. Surgery 1986;99(01):26-35

4 Moran SL, Illig KA, Green RM, Serletti JM. Free-tissue transfer in patients with peripheral vascular disease: a 10-year experience. Plast Reconstr Surg 2002;109(03):999-1006

5 Kucan JO, Robson MC. Diabetic foot infections: fate of the contralateral foot. Plast Reconstr Surg 1986;77(03):439-441

6 Toursarkissian B, Shireman PK, Harrison A, D’Ayala M, Schoolfield J, Sykes MT. Major lower-extremity amputation: contemporary experience in a single Veterans Affairs institution. Am Surg 2002; 68(07):606-610

7 Schofield CJ, Libby G, Brennan GM, MacAlpine RR, Morris AD, Leese GP; DARTS/MEMO Collaboration. Mortality and hospitalization in patients after amputation: a comparison between patients with and without diabetes. Diabetes Care 2006;29(10):2252-2256

8 Evans KK, Attinger CE, Al-Attar A, et al. The importance of limb preservation in the diabetic population. J Diabetes Complications 2011;25(04):227-231

9 Lin CW, Hsu BR, Tsai JS, et al. Effect of limb preservation status and body mass index on the survival of patients with limb-threatening diabetic foot ulcers. J Diabetes Complications 2017;31(01):180-185

10 Thorud JC, Plemmons B, Buckley CJ, Shibuya N, Jupiter DC. Mortality after nontraumatic major amputation among patients with diabetes and peripheral vascular disease: a systematic review. J Foot Ankle Surg 2016;55(03):591-599

11 DeFazio MV, Han KD, Iorio ML, et al. Combined free tissue transfer for the management of composite Achilles defects: functional outcomes and patient satisfaction following thigh-based vascularized reconstruction with a neotendon construct. J Reconstr Microsurg 2014;30(06):431-440

12 DeFazio MV, Han KD, Akbari CM, Evans KK. Free tissue transfer after targeted endovascular reperfusion for complex lower extremity reconstruction: setting the stage for success in the presence of mutlivessel disease. Ann Vasc Surg 2015;29(06):1316.e7-1316.e15

13 Driver VR, Fabbi M, Lavery LA, Gibbons G. The costs of diabetic foot: the economic case for the limb salvage team. J Vasc Surg 2010;52(3, Suppl):17S-22S

14 Hicks CW, Selvarajah S, Mathioudakis N, et al. Trends and determinants of costs associated with the inpatient care of diabetic foot ulcers. J Vasc Surg 2014;60(05):1247-1254, 1254.e1-1254.e2

15 Fitzgerald O'Connor EJ, Vesely M, Holt PJ, Jones KG, Thompson MM, Hinchliffe RJ. A systematic review of free tissue transfer in the management of non-traumatic lower extremity wounds in patients with diabetes. Eur J Vasc Endovasc Surg 2011;41(03):391-399

16 Sato T, Yana Y, Ichioka S. Free flap reconstruction for diabetic foot limb salvage. J Plast Surg Hand Surg 2017;51(06):399-404

17 Ozkan O, Coşkunfirat OK, Ozgentaş HE. Reliability of free-flap coverage in diabetic foot ulcers. Microsurgery 2005;25(02): 107-112

18 Eskelinen E, Kaartinen I, Kääriäinen M, Kuokkanen H. Successful foot salvage with microvascular flaps in diabetic patients. Scand J Surg 2015;104(02):103-107

19 Hong JP. Reconstruction of the diabetic foot using the anterolateral thigh perforator flap. Plast Reconstr Surg 2006;117(05):1599-1608

20 Meyer A, Goller K, Horch RE, et al. Results of combined vascular reconstruction and free flap transfer for limb salvage in patients with critical limb ischemia. J Vasc Surg 2015;61(05):1239-1248

21 Economides JM, Patel KM, Evans KK, Marshall E, Attinger CE. Systematic review of patient-centered outcomes following lower extremity flap reconstruction in comorbid patients. J Reconstr Microsurg 2013;29(05):307-316

22 Wang YC, Hart DL, Stratford PW, Mioduski JE. Clinical interpretation of a lower-extremity functional scale-derived computerized adaptive test. Phys Ther 2009;89(09):957-968

23 Falola RA, Lakhiani C, Green J, et al. Assessment of function after free tissue transfer to the lower extremity for chronic wounds using the lower extremity functional scale. J Reconstr Microsurg 2018;34(05):327-333

24 Nehler MR, Coll JR, Hiatt WR, et al. Functional outcome in a contemporary series of major lower extremity amputations. J Vasc Surg 2003;38(01):7-14

25 Attinger CE, Brown BJ. Amputation and ambulation in diabetic patients: function is the goal. Diabetes Metab Res Rev 2012;28 (Suppl 1):93-96 